\title{
Quantum information entropies of the eigenstates and the coherent state of the Pöschl-Teller potential
}

\author{
Rajneesh Atre ${ }^{1,2 *}$, Anil Kumar ${ }^{3}$, C. Nagaraja Kumar ${ }^{\dagger 3}$ \\ and \\ Prasanta K. Panigrahi $\ddagger 1,2$ \\ 1 Physical Research Laboratory, Navrangpura, Ahmedabad-380 009, India \\ 2 School of Physics, University of Hyderabad, Hyderabad-500 046, India \\ 3 Department of Physics, Panjab University, Chandigarh-160 014, India
}

The position and momentum space information entropies, of the ground state of the Pöschl-Teller potential, are exactly evaluated and are found to satisfy the bound, obtained by Beckner, Bialynicki-Birula and Mycielski. These entropies for the first excited state, for different strengths of the potential well, are then numerically obtained. Interesting features of the entropy densities, owing their origin to the excited nature of the wave functions, are graphically demonstrated. We then compute the position space entropies of the coherent state of the Pöschl-Teller potential, which is known to show revival and fractional revival. Time evolution of the coherent state reveals many interesting patterns in the space-time flow of information entropy.

\footnotetext{
*atre@prl.ernet.in

† cnkumar@pu.ac.in

$\ddagger$ prasanta@prl.ernet.in
} 


\section{INTRODUCTION}

Information entropy plays a crucial role in a stronger formulation of the uncertainty relations. The information theoretic uncertainty relations were first conjectured by Everett [1] and Hirschman [2] in 1957, and proved by Bialynicki-Birula and Mycielski, and independently by Beckner [3]. From the general properties of the Fourier transform, it was proved that, $S_{\text {pos }}+S_{m o m} \geq 1+\ln \pi$. Here, $S_{\text {pos }}=-\int d x|\psi(x)|^{2} \ln |\psi(x)|^{2}$ and $S_{m o m}=-\int d p|\tilde{\psi}(p)|^{2} \ln |\tilde{\psi}(p)|^{2}$, are the position and momentum space entropies, respectively. In a $D$-dimensional space, the right hand side of the above inequality contains a multiplicative factor $D$. The above equation, known in the literature as Beckner, BialynickiBirula and Mycielski (BBM) inequality, captures the physical fact that localized $|\psi(x)|^{2}$ leads to a diffused $|\tilde{\psi}(p)|^{2}$ and vice-versa. It should be emphasized that, though $S_{\text {pos }}$ and $S_{m o m}$ are individually unbounded, their sum is bounded from below. It is interesting to point out that, the above mentioned inequality was discovered by Everett in the context of many worlds interpretation of quantum mechanics. A general framework for deriving uncertainty relations of the above type, between general dynamical variables, not necessarily canonically conjugate ones, have been given recently [4-9].

These entropies lead to new and stronger version of the Heisenberg uncertainty relations. Using a variational inequality, relating entropy and standard deviation for an arbitrary one dimensional variable $A[3,10,11]: S(A) \leq \frac{1}{2}+\ln (\sqrt{2 \pi} \Delta A)$ and the BBM inequality, one can derive Heisenberg type uncertainty relations. These relations, which are based on standard deviations, are not very reliable, particularly when conjugate variables are discrete and the corresponding Hilbert space is finite dimensional. These entropies have been quite useful for characterizing quantum entanglement, since the von Neumann entropy $S=-\operatorname{Tr}\{\hat{\rho} \ln \hat{\rho}\}$, for the reduced density operators, is often difficult to calculate. Various properties of quantum mechanical entropy and its classical counterparts have been elucidated in Refs.[12-14]. As is clear, the single-particle distribution densities, measuring the spread of the wave functions in coordinate and momentum spaces, define their respective entropies. Interestingly, in the density functional theory of Hohenberg and Kohn, the single particle densities also completely characterize a many-body system [15].

The analytical determination of position and momentum space entropies have been carried out only for a few quantum mechanical systems. For the simple harmonic oscillator, 
the entropies were exactly calculated for the ground state, in both, coordinate and momentum space, for which the BBM inequality is saturated [16]. For an arbitrary state, the entropies were determined approximately, using asymptotic values of the entropy of the orthogonal Hermite polynomials. The entropy integrals $\int P_{n}^{2}(x) \ln P_{n}^{2}(x) d \mu(x)$, for several orthogonal polynomials $P_{n}$ 's having suitable measures, have been recently studied, from which the asymptotic expressions for the information entropies for large values of $n$, have been obtained for $D$ dimensional harmonic oscillator and Coulomb problems [17]. Information entropy of neutral atoms [18, 19], in the Thomas-Fermi theory, also manifests in a universal form, analogous to the one given in Ref. [3]. Information entropies in various contexts e.g., mathematical physics, mathematics, information theory, chemical physics and other areas of physics, have been extensively analyzed in recent times [20-36].

The present article is devoted to the study of the information entropies of the PöschlTeller (PT) family of potentials. These potentials widely appear in the analysis of soliton bearing nonlinear equations e.g., Bose-Einstein condensates and in quantum problems on curved background [37-39]. In the following section, we first consider the hyperbolic PT potential and evaluate the position and momentum space entropies exactly for the ground state, for a range of potential strengths. For the first excited state, we calculate these entropies, numerically, which is shown to satisfy the bound obtained by Bialynicki-Birula and Mycielski. Some interesting features of the entropy densities are then graphically demonstrated. In the third section, we compute the position space entropy densities of the coherent states of the trigonometric PT potential, which exhibits revival and fractional revival, due to interference effects. Under time evolution these densities reveal interesting patterns in the space-time flow of information entropy. We conclude in the last section after pointing out various future directions of work in this area.

\section{INFORMATION ENTROPY FOR PÖSCHL-TELLER SYSTEMS}

We begin with the Schrödinger equation for hyperbolic Pöschl-Teller potential, which is reflectionless and admits $n$ bound states for integer values of $n$ (In units $\hbar=2 m=1$ ),

$$
\left[-\frac{d^{2}}{d x^{2}}-\frac{n(n+1)}{4} \operatorname{sech}^{2}\left(\frac{x}{2}\right)\right] \psi^{(n)}(x)=E \psi^{(n)}(x) .
$$


The normalized ground state eigenfunction is given by,

$$
\psi_{o}^{(n)}(x)=\frac{1}{\sqrt{2 B\left(\frac{1}{2}, n\right)}} \cdot \operatorname{sech}^{n}\left(\frac{x}{2}\right)
$$

where $B\left(\frac{1}{2}, n\right)$ is the Beta-function.

Using the definition of position space entropy, after a lengthy but straightforward calculation, we obtain the analytical expression:

$$
S_{p o s}=-(2 n-1) \ln 2+\ln B\left(\frac{1}{2}, n\right)+2 n[\Psi(2 n)-\Psi(n)]
$$

where $\Psi$ is the digamma function.

For $n=1$ and 2 , the position space entropy has the values $S_{p o s}=2$ and $\frac{10}{3}-\ln 6$, respectively.

The corresponding momentum space entropies can be evaluated by first obtaining the momentum space ground state wavefunctions, which are the Fourier transforms of the corresponding position space wavefunctions:

$$
\psi_{0}^{(n)}(p)=A 2^{n} B\left(\frac{n}{2}+i p, \frac{n}{2}-i p\right)
$$

For $n=1, \psi_{o}(p)=\sqrt{\frac{\pi}{2}} \operatorname{sech}(\pi p)$ and the $S_{m o m}$ can be easily evaluated,

$$
\begin{aligned}
S_{m o m} & =-\int_{-\infty}^{\infty} 2 \psi_{o}^{2}(p) \ln \psi_{o}(p) d p \\
& =2-\ln (2 \pi)
\end{aligned}
$$

and the corresponding BBM inequality reads

$$
S_{\text {pos }}+S_{\text {mom }}=4-\ln 2 \pi \geq 1+\ln (\pi)
$$

For higher values of $n$, evaluation of momentum space entropies is quite cumbersome, instead we plot entropy-densities for both position and momentum space. As shown in Fig.1 and 2 , it is interesting to notice that, the position entropy-density plots, develop a dip at its peak as we increase the value of the parameter $n$; exactly contrary behavior is observed in their momentum space counterparts. As seen in Fig3., for the ground state, as $n$ increases, the BBM inequality tends to be saturated. Physically, for increasing $n$, the depth of the potential increases and it increasingly resembles the oscillator potential, which saturates the above inequality. 

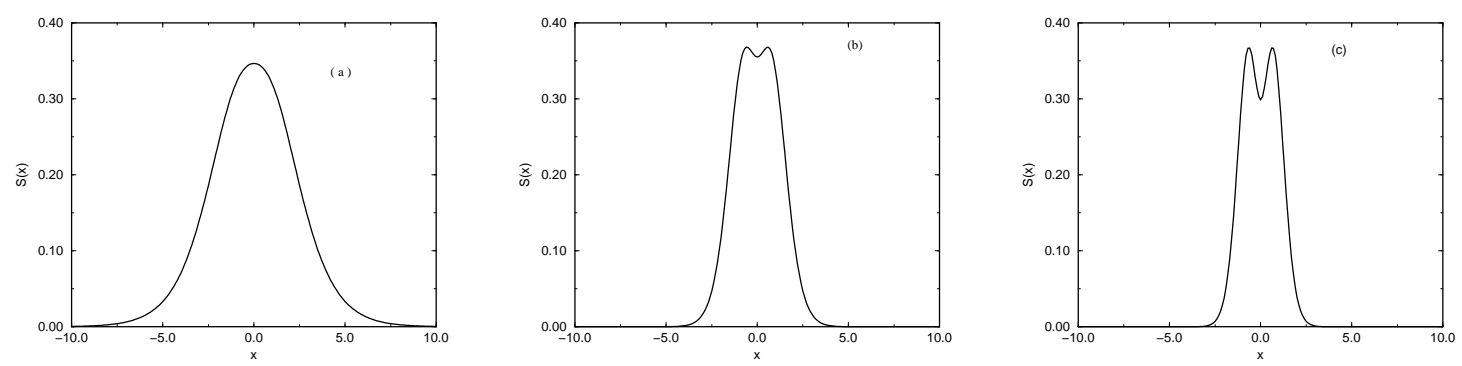

FIG. 1: Plots of the position space entropy densities for the ground state of hyperbolic Pöschl-Teller potential for (a) $n=1$, (b) $n=3$ and (c) $n=5$.
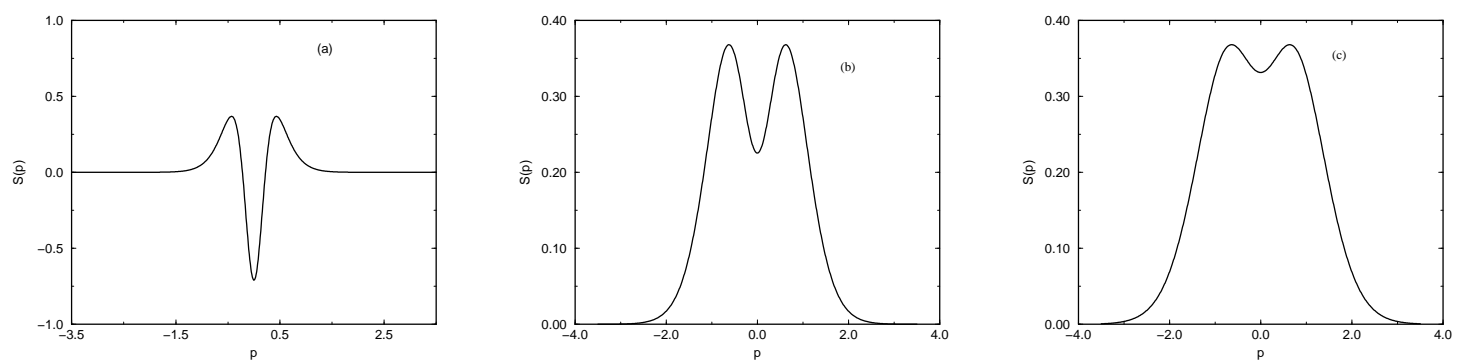

FIG. 2: Plots of the momentum space entropy densities for the ground state of hyperbolic PöschlTeller potential for (a) $n=1$, (b) $n=3$ and (c) $n=5$.

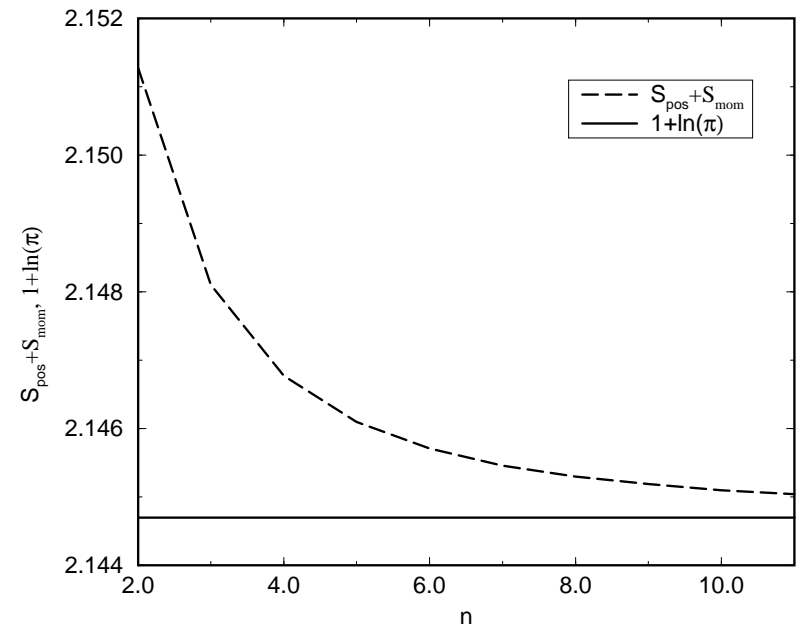

FIG. 3: The Plot depicting the variation of sum of position and momentum space entropies with respect to potential parameter $n$. 
We now proceed to evaluate the position space entropies of the first excited state. The corresponding wave function for the potential $V(x)$ reads,

$$
\psi_{1}^{(n)}(x)=N \operatorname{sech}^{n-1}\left(\frac{x}{2}\right) \tanh \left(\frac{x}{2}\right) \quad \text { for } \quad n \geq 2
$$

where $N=\frac{1}{\sqrt{2\left[B\left(\frac{1}{2}, n-1\right)-B\left(\frac{1}{2}, n\right)\right]}}$ is the normalization constant.

For $n=2, S_{\text {pos }}=2.23472$ and for general $n$ the behavior of the position space information entropy is depicted in Fig.2. Table.1, depicts the BBM inequality for the first excited state as a function of $n$. One sees that, as the value of $n$ increases the sum of the entropies tends towards a saturation value higher than the ground state value.

Table 1: Table for BBM inequality for the first excited state of the hyperbolic Pöschl-Teller potential.

\begin{tabular}{|c|c|c|c|c|c|c|c|c|c|}
\hline$n$ & $S_{\text {pos }}$ & $S_{m o m}$ & $S_{\text {pos }}+S_{\text {mom }}$ & $1+\ln \pi$ & $n$ & $S_{\text {pos }}$ & $S_{m o m}$ & $S_{p o s}+S_{m o m}$ & $1+\ln \pi$ \\
\hline 2 & 2.23472 & 0.722555 & 2.95728 & 2.1447 & 8 & 1.0971 & 1.63508 & 2.73217 & 2.1447 \\
\hline 3 & 1.7988 & 1.0384 & 2.8372 & 2.1447 & 9 & 1.02621 & 1.70025 & 2.72646 & 2.1447 \\
\hline 4 & 1.56242 & 1.22799 & 2.7904 & 2.1447 & 10 & 0.96409 & 1.7579 & 2.72199 & 2.1447 \\
\hline 5 & 1.40082 & 1.36474 & 2.76556 & 2.1447 & 11 & 0.908807 & 1.80958 & 2.71839 & 2.1447 \\
\hline 6 & 1.27825 & 1.47193 & 2.75018 & 2.1447 & 12 & 0.859009 & 1.85643 & 2.71544 & 2.1447 \\
\hline 7 & 1.1796 & 1.56013 & 2.73973 & 2.1447 & 13 & 0.81371 & 1.89926 & 2.71297 & 2.1447 \\
\hline
\end{tabular}

\section{ENTROPY DENSITIES FOR COHERENT STATES OF TRIGONOMETRIC PÖSCHL-TELLER POTENTIAL}

Quantum systems with eigen spectra depending quadratically on a quantum number $n$ are known to show revival and partial revivals in time evolution of corresponding wave packets. These quantum carpet structures have been studied quite extensively [40]. There have been 
suggestions to use the revival structure for obtaining a factorization algorithm [41]. The possibility of realizing PT type of potentials in atomic systems such as BEC, through optical means, makes the study of time evolution of these systems more interesting [42]. It should be pointed out that perturbation of BEC on a soliton or cnoidal wave type solitary train background are known to satisfy the hyperbolic or trigonometric PT Schrödinger equations [38]. In the following we study the time evolution of the information entropy density for an annihilation operator coherent state of the trigonometric PT potential [43]. The fact that, coherent structure like laser is an annihilation operator eigen state and the coherent manipulation of atoms, possibly with optical means is being increasingly considered seriously, may make these analyses useful. The trigonometric case has been chosen deliberately, since it has an infinite number of bound states as compared to the hyperbolic one, which makes the construction of the coherent states straightforward.

We consider here the Hamiltonian of symmetric Pöschl-Teller(SPT) potential (in the units $\hbar=2 m=1$ ),

$$
H=-\frac{d^{2}}{d y^{2}}+\frac{\alpha^{2} \rho(\rho-1)}{\cos ^{2}(\alpha y)}
$$

with eigenvalues and eigenfunctions, in the variable $x=\sin (\alpha y)$,

$$
\begin{aligned}
E_{n}^{S P T} & =\alpha^{2}(n+\rho)^{2} \\
\psi_{n}^{S P T}(x) & =\left[\frac{\alpha(n !)(n+\rho) \Gamma(\rho) \Gamma(2 \rho)}{\sqrt{\pi} \Gamma(\rho+1 / 2) \Gamma(n+2 \rho)}\right]^{1 / 2}\left(1-x^{2}\right)^{\rho / 2} C_{n}^{\rho}(x) .
\end{aligned}
$$

Recently based on a dynamical SU(1,1) algebra, an annihilation operator coherent state, was constructed for this system: $K_{-}|\gamma>=\gamma| \gamma>$, here $K_{-}$is the annihilation operator of the $\mathrm{SU}(1,1)$ algebra [43]. The coordinate space realization of this coherent state is given by:

$$
\tilde{\chi}_{S P T}(x, \gamma)=N(\gamma)^{-1} \sum_{n=0}^{\infty}\left[\frac{\Gamma(2 \rho) \Gamma(\rho+1 / 2) \sqrt{\pi}}{\alpha(n !)(n+\rho) \Gamma(2 \rho) \Gamma(2 \rho+n)}\right]^{1 / 2} \gamma^{n} \psi_{n}^{S P T}(x)
$$

As already pointed out in the beginning of this section, the quadratic nature of the spectra of SPT potential leads to the possibility of revival and fractional revival in this quantum system due to subtle interference effects. Keeping in mind, the fact that in the realistic situations the complete span of the wave functions may not be available, we study the time evolution of position space entropy densities for various values of $n$, i.e., the number of states, interfering and constituting the resultant coherent wave packet. The effect of change 

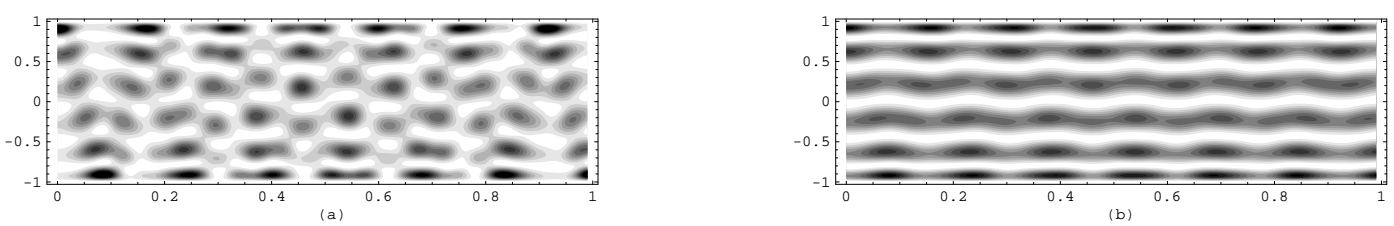

FIG. 4: Contour plots, depicting time evolution of position space entropy densities for the coherent states trigonometric Pöschl-Teller potential for (a) $n=5, \gamma=10$ and (b) $n=5, \gamma=30$. Darkness displays a low and brightness a high functional value.
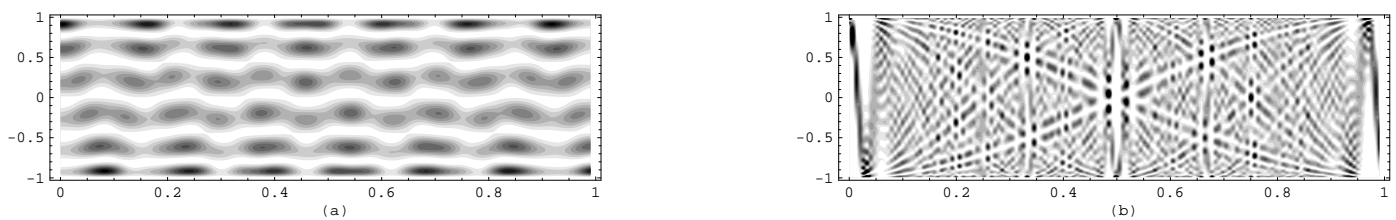

FIG. 5: Contour plots, depicting time evolution of position space entropy densities for the coherent states trigonometric Pöschl-Teller potential for (a) $n=5, \gamma=15$ and (b) $n=30, \gamma=15$. Darkness displays a low and brightness a high functional value.

of coherence parameter $\gamma$ on the same is also analyzed. These are depicted in the Figs. 4 and 5. One finds dramatic changes in the carpet structure of the entropy densities in space and time. One observes rich tapestry like structures, where one can manipulate the valleys and ridges of the entropy density in space and time. It is interesting to observe that, as we increase the value of coherence parameter $\gamma$, keeping $n$ fixed at some value, the various ridges come close together and form a continuous structure. These patterns become sharper for the higher values of $n$.

\section{CONCLUSIONS}

In conclusion, we have studied the information entropies of a class of quantum systems belonging to the Pöschl-Teller family of potentials. Exact results, for the position space entropies of the ground and first excited states of the hyperbolic Pöschl-Teller potential were obtained, for a range of potential strengths. The expression for momentum space entropy was obtained analytically for the ground state and numerically computed for the first excited state. It was found that, these entropies satisfy the Beckner, Bialynicki-Birula and Mycielski inequality. The entropy densities for the above cases were depicted graphically, 
for demonstrating the entropy distribution in the well. For the trigonometric case, after investigating the entropies associated with the eigenstates, we studied the time evolution of entropy density for the coherent state [43]. The intricate carpet structure shows the richness of this quantum system, which needs to be explored further. It should be noted that, coherent states are being envisaged for the storage of quantum information. Pöschl-Teller potential manifests in quantum problems on curved background [39], as also in non-linear integrable models with soliton solutions like, KdV equation [37]. In light of this, the physical relevance of the information entropies computed here, needs further study. We hope to come back to some of these questions in future.

Acknowledgements: We acknowledge many useful discussions with Prof. K.D. Sen, who also brought to our notice many relevant references. We are thankful to Prof. V.B. Sheorey for discussions and to Dr. J. Banerji for help in the numerical works. A.K. acknowledges the financial support of CSIR India through S.R.F.

[1] H. Everett, The Many-Worlds Interpretation of Quantum Mechanics (Princeton, NJ: Princeton University Press, 1973).

[2] I.I. Hirschman, Am. J. Math. 79, 152 (1957).

[3] W. Beckner, Ann. Math. 102, 159 (1975);

I. Bialynicki-Birula and J. Mycielski, Comm. Math. Phys. 44, 129 (1975).

[4] D. Deutsch, Phys. Rev. Lett. 50, 631 (1983).

[5] M.H. Partovi, Phys. Rev. Lett. 50, 1883 (1983).

[6] I. Bialynicki-Birula, Phys. Lett. 103A, 253 (1984).

[7] V.V. Dodonov and V.I. Manko, Generalizations of uncertainty relations in quantum mechanics, Lebedev Institute Papers, 183, 5 (1987).

[8] H. Massen and J.B.M. Uffink, Phys. Rev. Lett. 60, 1103 (1988).

[9] J. Sánchez-Ruiz, Phys. Lett. A 181, 193 (1993).

[10] C.E. Shannon, Bell Systems Technol. J. 27, 379, 623 (1948).

[11] J.S. Dehesa, A. Martínez-Finkelshtein and J. Sánchez-Ruiz, J. Comput. Appl. Math. 133, 23 (2001).

[12] A. Wehrl, Rev. Mod. Phys. 50, 221 (1978). 
[13] A. Wehrl, Rep. Math. Phys. 16, 353 (1979).

[14] D. Petz, Entropy, von Neumann and von Neumann Entropy, quant-ph/010201.

[15] P. Hohenberg and W. Kohn, Phys. Rev. 136, B864 (1964); W. Kohn and L.J. Sham, ibid. 140, A1133 (1965).

[16] V. Majerník and T. Opatrný, J. Phys. A 29, 2187 (1996).

[17] R.J. Yáñez, W. Van Assche and J.S. Dehesa, Phys. Rev. A 50, 3065 (1994).

[18] S.R. Gadre, Phys. Rev. A 30, 620 (1983).

[19] S.R. Gadre, S.B. Sears, S.J. Chakravorty and R.D. Bendale, Phys. Rev. A 32, 2602 (1985).

[20] S. Abe and N. Suzuki, Phys. Rev. A 41, 4608 (1990).

[21] V. Majerník and L. Richterek, J. Phys. A 30, L49 (1997).

[22] V. Majerník and L. Richterek, Eur. J. Phys. 18, 79 (1997).

[23] M.S. Santhanam, V.B. Sheorey and A. Lakshminarayan, Phys. Rev. E 57, 345 (1998).

[24] V.S. Buyarov, J.H. Dehesa and A. Martínez-Finkelshtein, J. Approx. Theo. 99, 153 (1999).

[25] M.S. Santhanam, Entropic uncertainty relations for the ground state of a coupled system, quant-ph/0308147.

[26] G.C. Ghirardi, L. Marinatto and R. Romano, Phys. Lett. A 317, 32 (2003).

[27] V.S. Buyarov, On information entropy of Gegenbauer polynomials, Vesnt. Mosk. Univ. (Ser. 1) $, 6,8$ (1997).

[28] V.S. Buyarov, P. López-Artés, A. Martínez-Finkelshtein and W. Van Assche, J. Phys. A 33, $6549(2000)$.

[29] N. Balakrishnan and N. Sathyamurthy, Chem. Phys. Lett. 164, 267 (1989).

[30] G.S. Agarwal and J. Banerji, Opt. Lett. 27, 800 (2002).

[31] S. Abe, Phys. Lett. A 166, 163 (1992).

[32] A.R. González, J.A. Vaccaro and S.M. Barnett, Phys. Lett. A 205, 247 (1995).

[33] S. Abe, S. Martínez, F. Pennini and A. Plastino, Phys. Lett. A 295, 74 (2002).

[34] M.W. Coffey, J. Phys. A 36, 7441 (2003).

[35] V. Ferrari and B. Mashhoon, Phys. Rev. D 30, 295 (1984).

[36] A.K. Rajagopal and S. Teitler, Phys. Lett. A 115, 313 (1986).

[37] A. Das, Integrable Models (World Scientific, Singapore, 1989).

[38] C. J. Pethick, and H. Smith, Bose-Einstein Condensation in Dilute Gases (Cambridge University Press, 2001) and references therein. 
[39] D.P. Jatkar, Nucl. Phys. B 395, 167 (1993);

E. Witten, Phys. Rev. D 44, 314 (1991).

[40] R. Bluhm, A. Kostelecky, J. Porter, Am. J. Phys. 64, 944 (1996).

[41] H. Mack, M. Bienert, F. Haug, M. Freyberger, W.P. Schleich Wave Packets can Factorize Numbers, quant-ph/0208021.

[42] B. Deconinck, J.N. Kutz, M.S. Patterson and B.W. Warner, J. Phys. A 36, 5431 (2003).

[43] T. Shreecharan, P.K. Panigrahi and J. Banerji, Coherent states for exactly solvable potentials, quant-ph/0309038, accepted for publication in Phys. Rev. A. 